12

\title{
Простые модели латеральных гетероструктур
}

\author{
() С.Ю. Давыдов
}

Физико-технический институт им. А.Ф. Иофрфе РАН,

Санкт-Петербург, Россия

E-mail: Sergei_Davydov@mail.ru

(Поступила в Редакцию 18 января 2018 г.)

Методом функций Грина в приближении сильной связи получены общие аналитические выражения для плотностей состояний латеральной гетероструктуры, образованной контактом двух квадратных полубесконечных решеток с однозонным и двухзонным спектрами. Для численных оценок использована полуэллиптическая плотность состояний, а для оценки перехода заряда предложена модель двух взаимодействующих димеров. Обсуждается применение настоящего подхода к описанию латеральных эпитаксиальных и графеноподобных гетероструктур.

DOI: 10.21883/FTT.2018.07.46129.015

\section{1. Введение}

Теоретическое исследование гетероструктур (ГС) на основе комбинаций химически различных двумерных (2D) графеноподобных соединений представляет непосредственный интерес для создания приборов наноэлектроники. В большинстве случаев речь идет о вертикальных структурах, когда 2D слои располагаются параллельно друг другу вдоль оси $z$, перпендикулярной к их плоскостям [1-4]. Такие структуры называют вандер-ваальсовыми, так как слои связаны дисперсионными силами. В меньшей степени внимание уделяется латеральным структурам, образованным состыкованными по кромкам 2D-слоями, расположенными в одной плоскости $[3,5,6]$. Отметим, что наиболее популярны ГС, где компонентами является графен и гексагональный нитрид бора $(h$-BN), причем как для вертикального $[4,7,8]$, так и для латерального $[6,9,10]$ вариантов.

В настоящей работе мы предложим схему описания электронной структуры латеральных ГС, основанную на методе функций Грина и теории сильной связи. При этом для простоты будут рассмотрены модели контактов одноатомных и двухатомных квадратных решеток с одинаковыми расстояниями между ближайшими соседями (б.c.), чтобы избежать усложнений, связанных со структурным рассогласованием.

\section{2. Полубесконечные 2D-решетки: метод Калкстейна-Совена}

Рассмотрим бесконечную одноатомную квадратную решетку, расположенную в плоскости $(x, y)$, с расстоянием $a$ между б.с. и законом дисперсии электронов $\varepsilon(\mathbf{k})=\varepsilon-2 t\left[\cos \left(k_{x} a\right)+\cos \left(k_{y} a\right)\right]$, где $\varepsilon-$ энергия атомной $s$-орбитали, $t$ - энергия перехода электрона между б.c., $\mathbf{k}=\left(k_{x}, k_{y}\right)$ - волновой вектор. Функция
Грина такой решетки имеет вид:

$$
G_{0}(\omega, \mathbf{k})=\frac{g(\omega)}{1+2 \operatorname{tg}(\omega)\left[\cos \left(k_{x} a\right)+\cos \left(k_{y} a\right)\right]} .
$$

Здесь $g^{-1}(\omega)=\omega-\varepsilon+i s-$ одноатомная функция Грина, $\omega-$ энергетическая переменная, $s=0^{+}[11,12]$. Такая одноатомная решетка представляет собой простейшую модель 2D-металла.

Аналогично, для бесконечной двухатомной квадратной решетки, состоящей из атомов $A$ и $B$, находящихся на расстоянии $a$ друг от друга, имеем $\varepsilon_{ \pm}(\mathbf{k})=\bar{\varepsilon} \pm \sqrt{\Delta^{2}+4 t^{2}\left[\cos ^{2}\left(k_{x} a\right)+\cos ^{2}\left(k_{y} a\right)\right]}, \quad$ где $\bar{\varepsilon}=$ $=\left(\varepsilon_{A}+\varepsilon_{B}\right) / 2, \Delta=\left(\varepsilon_{A}-\varepsilon_{B}\right) / 2$. Соответствующая функция Грина есть

$G_{0}^{A(B)}(\omega, \mathbf{k})=\frac{g_{A(B)}(\omega)}{1-4 g_{A}(\omega) g_{B}(\omega) t^{2}\left[\cos ^{2}\left(k_{x} a\right)+\cos ^{2}\left(k_{y} a\right)\right]}$,

где $g_{A(B)}^{-1}(\omega)=\omega-\varepsilon_{A(B)}+i s, \varepsilon_{A(B)}-$ энергия $s$-орбитали атома $A(B)[11,12]$. Такая двухатомная решетка является простейшей моделью $2 \mathrm{D}$-полупроводника с шириной запрещенной зоны $E_{g}=2|\Delta|$.

Для перехода к полубесконечным решеткам, что необходимо для нахождения соответствующих функций Грина $G$ и $G^{A(B)}$, воспользуемся подходом Калкстейна и Совена [13]. Пусть бесконечной решетке отвечает гамильтониан $H_{0}$, а полубесконечной - гамильтониан $H=H_{0}+T$, где $T-$ возмущение, описывающее разрезание (разрыв межатомных связей) бесконечной решетки, в результате чего возникают левая $(L)$ и правая $(R)$ полубесконечные решетки. Обозначая через $|\mathbf{R}\rangle$ локализованную на узле решетки $\mathbf{R}=(x, y)$ функцию Ваннье, можно записать

$$
\left\langle\mathbf{R}|T| \mathbf{R}^{\prime}\right\rangle=-\left\langle\mathbf{R}\left|H_{0}\right| \mathbf{R}^{\prime}\right\rangle,
$$

где $|\mathbf{R}\rangle \in R,\left|\mathbf{R}^{\prime}\right\rangle \in L$. Соотношение (3) следует из того простого факта, что после разрезания правая и левая 
половины исходной решетки не связаны между собой, т. е. $\left\langle\mathbf{R}|H| \mathbf{R}^{\prime}\right\rangle=0$.

Будем считать, что ось $x$ перпендикулярна, а ось $y$ параллельна разрезу. Тогда для бесконечной одноатомной решетки справедливы трансляционные соотношения вида $\left\langle m, k_{y}\left|G_{0}\right| n, k_{y}^{\prime}\right\rangle=\delta\left(k_{y}-k_{y}^{\prime}\right) G_{0}\left(\omega, m-n, k_{y}\right)$, $\left\langle m, k_{y}|G| n, k_{y}^{\prime}\right\rangle=\delta\left(k_{y}-k_{y}^{\prime}\right) G\left(\omega, m, n, k_{y}\right)$ и $\left\langle m, k_{y}|T| n, k_{y}^{\prime}\right\rangle=$ $=\delta\left(k_{y}-k_{y}^{\prime}\right) T(m, n)$, где $m(n)-$ номера атомных рядов, так что $x_{m(n)}=m a(n a)$. Для двухатомной решетки можно записать аналогичные соотношения, заменив $m a(n a)$ на $2 m a(2 n a)$. Считаем, что $m \geq 0$ соответствуют области $R, m \leq-1$ отвечает области $L$. При этом отличными от нуля являются только матричные элементы $T(-1,0)$ и $T(0,-1)$, равные по модулю $t^{1}$.

Начнем с одноатомной решетки. Воспользовавшись уравнением Дайсона $G=G_{0}+G_{0} T G$, получим для функции Грина правой полубесконечной решетки $(m \geq 0)$ следующее выражение:

$$
G(m, m)=G_{0}(0)+t G_{0}(m+1) G_{0}(-m)\left[1-t G_{0}(1)\right]^{-1}
$$

На разрезе „Поверхностная“ функция Грина (4) имеет вид

$$
G(0,0)=\frac{G_{0}(0)}{1-t G_{0}(1)} .
$$

Для левой полубесконечной решетки $(m \leq-1)$ вместо выражений (4) и (5) получим соответственно

$$
\begin{gathered}
G(m, m)=G_{0}(0)+t G_{0}(-m-1) G_{0}(m)\left[1-t G_{0}(-1)\right]^{-1}, \\
G(-1,-1)=\frac{G_{0}(0)}{1-t G_{0}(-1)} .
\end{gathered}
$$

В формулах (4)-(7) в аргументах функций Грина опущены переменные $\omega$ и $k_{y}$.

Воспользовавшись результатами п. 1 Приложения, для случая $\alpha^{2}=4 t^{2} / W^{2}<1$, где $W=\Omega+2 t \cos \left(k_{y} a\right)$, $\Omega=\omega-\varepsilon$ легко показать, что

$$
G(-1,-1)=G(0,0) \equiv G\left(\omega, k_{y}\right)=\frac{2}{W} \frac{1-\sqrt{1-\alpha^{2}}}{\alpha^{2}},
$$

или $G\left(\omega, k_{y}\right)=\left(W-\sqrt{W^{2}-4 t^{2}}\right) / 2 t^{2}$. Так как в этом случае функция Грина не содержит мнимой части, соответствующая плотность состояний $\rho\left(\omega, k_{y}\right)=-\pi^{-1} \operatorname{Im} G\left(\omega, k_{y}\right)=0 . \quad$ При $\quad \alpha^{2}>1$ нужно заменить $\sqrt{1-\alpha^{2}}$ на $i \sqrt{\alpha^{2}-1}$. Отсюда следует, что $\quad G\left(\omega, k_{y}\right)=\left(W-i \sqrt{4 t^{2}-W^{2}}\right) / 2 t^{2}$. Тогда соответствующая плотность состояний $\left.\rho\left(\omega, k_{y}\right)=\sqrt{4 t^{2}-W^{2}}\right) / 2 \pi t^{2}$.

Перейдем теперь к случаю двухатомной квадратной решетки. Выражения для функций Грина $G_{0}^{A(B)}(m)$ приведены в Приложении, п. 1. Для „Поверхностных“ функций Грина $G_{0}^{A(B)}$ полубесконечных решеток справедливы

\footnotetext{
1 Здесь мы не вводим в матричные элементы множители вида $\exp \left(i \vartheta\left(k_{y}\right)\right)$, так как они не существенны для произведения $T(-1,0) T(0,-1)=t^{2}$. Поэтому, не теряя общности, можно положить $T(-1,0)=T(0,-1)=t$.
}

формулы (5), (7) и (8) с заменой $G_{0}, G$ и $\alpha^{2}$ на $G_{0}^{A(B)}$, $G^{A(B)}$ и $\beta^{2}$.

\section{3. Гетероконтакты: общие соотношения}

3.1. Контакт одноатомных решеток. Начнем с простейшего случая ГС, образованной состыковкой двух полубесконечных одноатомных решеток: левой $(L)$ с $m \leq-1$ и правой $(R)$ с $m \geq 0$, различающихся только значениями энергий атомных $s$-орбиталей, равными соответственно $\varepsilon_{L}$ и $\varepsilon_{R}$. Эти решетки, характеризуемые для простоты одинаковым расстоянием $a$ между б.с., связаны между собой матричными элементами $\tilde{T}(-1,0)=\tilde{T}(0,-1)=\tilde{t}$. Такая система моделирует контакт двух 2D-металлов. Ниже в функциях Грина мы опускаем аргументы $\omega$ и $k_{y}$, знак „тильда“ относится к ГС.

Воспользовавшись уравнением Дайсона $\tilde{G}=G+G \tilde{T} \tilde{G}$, получим

$$
\begin{array}{r}
\tilde{G}(-1,-1)=G(-1,-1)+G(-1,-1) \tilde{T}(-1,0) \tilde{G}(0,-1), \\
\tilde{G}(0,-1)=G(0,0) \tilde{T}(0,-1) \tilde{G}(-1,-1),
\end{array}
$$

откуда

$$
\tilde{G}(-1,-1)=G(-1,-1)\left[1-\tilde{t}^{2} G(-1,-1) G(0,0)\right]^{-1} .
$$

Аналогичным образом найдем

$$
\tilde{G}(0,0)=G(0,0)\left[1-\tilde{t}^{2} G(-1,-1) G(0,0)\right]^{-1} .
$$

Обозначив $\quad G(-1,-1) \equiv G_{L}, \quad G(0,0) \equiv G_{R} \quad$ и $\tilde{G}(-1,-1) \equiv \tilde{G}_{L}, \tilde{G}(0,0) \equiv \tilde{G}_{R}$, из выражений $(13),(14)$ получим

$$
\tilde{G}_{L(R)}\left(\Omega_{L(R)}\right)=\frac{G_{L(R)}\left(\Omega_{L(R)}\right)}{1-\tilde{t}^{2} G_{L}\left(\Omega_{L}\right) G_{R}\left(\Omega_{R}\right)},
$$

где $\Omega_{L(R)}=\omega-\varepsilon_{L(R)} . \quad$ Положим $\quad\left(1-\tilde{t}^{2} G_{L} G_{R}\right)^{-1} \equiv$ $\equiv C=\left(G_{+}+G_{-}\right) / 2$, где $G_{ \pm}^{-1}=1 \pm \tilde{t} \sqrt{G_{L} G_{R}}$. Переходя в случае $W_{L(R)}^{2} \leq 4 t^{2}$ к комплексному представлению функций Грина $G_{L(R)}=t^{-1} \exp \left(i \varphi_{L(R)}\right)$, где $\varphi_{L(R)}=$ $=\operatorname{Arctg}\left(-\sqrt{4 t^{2}-W_{L(R)}^{2}} / W_{L(R)}\right) \quad$ и $\quad W_{L(R)}=\Omega_{L(R)}+$ $+2 t \cos \left(k_{y} \alpha\right)$, получим

$$
G_{ \pm}=\frac{1 \pm \tau \cos \phi \mp i \tau \sin \phi}{1 \pm 2 \tau \cos \phi+\tau^{2}}
$$

где безразмерная константа связи $\tau=\tilde{t} / t$ и $\phi=\left(\varphi_{L}+\varphi_{R}\right) / 2$. Плотности состояний, соответствующие функциям Грина (15), равны

$$
\tilde{\rho}_{L(R)}=-(\pi t)^{-1}\left(\cos \varphi_{L(R)} \operatorname{Im} C+\sin \varphi_{L(R)} \operatorname{Re} C\right),
$$

где $\quad \operatorname{Re} C=\left(1-\tau^{2} \cos 2 \phi\right) / A, \quad \operatorname{Im} C=\tau^{2} \sin 2 \phi / A \quad$ и $A=1-2 \tau^{2} \cos 2 \phi+\tau^{4}$. Легко показать, что при 
$W_{L(R)}^{2} \leq 4 t^{2} \quad$ имеем $\quad \sin \varphi_{L(R)}=-\sqrt{4 t^{2}-W_{L(R)}^{2}} / 2 t \quad$ и $\cos \varphi_{L(R)}=W_{L(R)} / 2 t$. В случае $W_{L(R)}^{2}>4 t$ в формуле (17) и аналогичных выражениях, приведенных ниже, следует формально положить $\sin \varphi_{L(R)}=0 \quad$ и $\cos \varphi_{L(R)}=\left(W_{L(R)}-\sqrt{W_{L(R)}^{2}-4 t^{2}}\right) / 2 t$. Отметим, что условия $W_{L(R)}^{2} \leq 4 t^{2} \quad$ и $\quad W_{L(R)}^{2}>4 t^{2} \quad$ эквивалентны неравенствам $\left|\Omega_{L(R)}\right| \leq 4 L$ и $\left|\Omega_{L(R)}\right|>4 t$. Некоторые характерные частные случаи рассмотрены в Приложении, П2.

3.2. Контакт одноатомной и двухатомной р еш е т о к. Перейдем теперь к ГС, образованной состыковкой полубесконечных левой одноатомной $(L, m \leq-1$, энергия орбитали $|s\rangle_{L}$ равна $\left.\varepsilon\right)$ и правой двухатомной $(R$, $m \geq 0$, энергии орбиталей $\left|s_{A}\right\rangle_{R}$ и $\left|s_{B}\right\rangle_{R}$ равны соответственно $\varepsilon_{A}$ и $\varepsilon_{B}$ ) полубесконечных квадратных решеток, для которых расстояние между б.с. равно $\alpha$. Матричные элементы связи орбиталей $|s\rangle_{L}$ и $\left|s_{A(B)}\right\rangle_{R}$ равны $\tilde{t}$. Такая ГС моделирует контакт 2D-металла и полупроводника с шириной запрещенной зоны $E_{g}=\left|\varepsilon_{A}-\varepsilon_{B}\right|$ (см. (2)).

Считая, что в узле 0 находится атом $A(B)$, можно повторить выкладки (12)-(14) и получить по аналогии c (15) четыре функции Грина вида

$$
\begin{gathered}
\tilde{G}_{L A^{\prime}(R A)}\left(\Omega_{L(R A)}\right)=\frac{G_{L(R A)}\left(\Omega_{L(R A)}\right)}{1-\tilde{t}^{2} G_{L}\left(\Omega_{L}\right) G_{R A}\left(\Omega_{R A}\right)}, \\
\tilde{G}_{L B^{\prime}(R B)}\left(\Omega_{L(R B)}\right)=\frac{G_{L(R B)}\left(\Omega_{L(R B)}\right)}{1-\tilde{t}^{2} G_{L}\left(\Omega_{L}\right) G_{R B}\left(\Omega_{R B}\right)},
\end{gathered}
$$

где $\Omega_{L}=\omega-\varepsilon, \Omega_{R A(B)}=\omega-\varepsilon_{A(B)}$; индекс $L A^{\prime}\left(B^{\prime}\right)$ у функций Грина $\tilde{G}_{L A^{\prime}(R A)}$ и $\tilde{G}_{L B^{\prime}(R B)}$ отвечает состоянию атома края $L$-решетки, взаимодействующего соответственно с атомами $A$ и $B$ края $R$-решетки. Таким образом, поставленная задача в нашей схеме сводится к двум независимым задачам о взаимодействии одноатомных полубесконечных решеток, рассмотренным в разделе 3.1, а именно: задаче об $L$-решетке, характеризуемой $s$-состоянием с энергией $\varepsilon$, связанной с $R$-решеткой, характеризуемой $s$-состояниями с энергиями $\varepsilon_{A}$ и $\varepsilon_{B}$. Поэтому все полученные в 3.1 выражения справедливы, если заменить нижние индексы: у функции Грина $\tilde{G}_{L}$ на $L A^{\prime}(R A)$ и $L B^{\prime}(R B)$, у функции Грина $\tilde{G}_{R}-$ на $R A$ и $R B$, у энергетических параметров $\Omega_{R}$ на $\Omega_{R A}, \Omega_{R B}$ и $\varepsilon_{R}$ на $\varepsilon_{A}, \varepsilon_{B}$ соответственно. Положив $\varepsilon_{A}+\varepsilon_{B}=0$, т.е. поместив начало отсчета энергии в середину запрещенной зоны 2D-полупроводника, и варьируя значение $\varepsilon$, получим различные варианты энергетической диаграммы системы. В соответствии с общим правилом $\tilde{\rho}_{L A^{\prime}(R A)}=\pi^{-1} \operatorname{Im} \tilde{G}_{L A^{\prime}(R A)}$ и $\tilde{\rho}_{L B^{\prime}(R B)}=\pi^{-1} \operatorname{Im} \tilde{G}_{L B^{\prime}(R B)}$.

3.3. Контакт двухатомных решеток. Рассмотрим, наконец, контакт двухатомных полубесконечных решеток, правая из которых (R-решетка) по-прежнему характеризуется энергиями $s$-орбиталей $\varepsilon_{A}$ и $\varepsilon_{B}$ атомов $A$ и $B$ соответственно $\left(E_{g R}=2 \Delta_{R}=\left|\varepsilon_{A}-\varepsilon_{B}\right|\right)$, а левая ( $L$-решетка) - энергиями $\varepsilon_{C}$ и $\varepsilon_{D}$, отвечающими $s$-орбиталям атомов $C$ и $D \quad\left(E_{g L}=2 \Delta_{L}=\left|\varepsilon_{C}-\varepsilon_{D}\right|\right)$. Будем считать, что между собой связаны атомы $A$ и $C, B$ и $D$, а матричные элементы связи равны $\tilde{t}$. Легко сообразить, что четыре соответствующих задаче функции Грина имеют вид

$$
\begin{gathered}
\tilde{G}_{C(A)}\left(\Omega_{C(A)}\right)=\frac{G_{C(A)}\left(\Omega_{C(A)}\right)}{1-\tilde{t}^{2} G_{C}\left(\Omega_{C}\right) G_{A}\left(\Omega_{A}\right)}, \\
\tilde{G}_{D(B)}\left(\Omega_{D(B)}\right)=\frac{G_{D(B)}\left(\Omega_{D(B)}\right)}{1-\tilde{t}^{2} G_{D}\left(\Omega_{D}\right) G_{B}\left(\Omega_{B}\right)},
\end{gathered}
$$

где $\Omega_{C(D)}=\omega-\varepsilon_{C(D)}, \Omega_{A(B)}=\omega-\varepsilon_{A(B)}$. Такая ГС отвечает гетеропереходу на контакте двух полупроводников. Варьируя значения параметров $\varepsilon_{A}, \varepsilon_{B}, \varepsilon_{C}$ и $\varepsilon_{D}$, можно получить 2D-аналоги объемных (см., например, [14]) и эпитаксиальных [15] ГС. Соответствующие плотности состояний равны $\tilde{\rho}_{C(A)}=\pi^{-1} \operatorname{Im} \tilde{G}_{C(A)}$ и $\tilde{\rho}_{D(B)}=\pi^{-1} \operatorname{Im} \tilde{G}_{D(B)}$.

\section{4. Полуэллиптические плотности состояний: оценки}

Все полученные выше функции Грина $\tilde{G}, G$ и плотности состояний $\tilde{\rho}, \rho$ зависят как от энергии $\omega$, так и от волнового вектора $k_{y}$. Для расчета электронного заполнения атомов $m$-го $y$-ряда атомов и перехода заряда между атомными рядами, параллельными линии контакта, необходимы зависящие только от энергии функции Грина $\tilde{G}(\omega), G(\omega)$ и плотности состояний $\tilde{\rho}(\omega)$, $\rho(\omega)$. Проинтегрировать точно выражение (8) по $k_{y}$ не представляется, однако, возможным. Поэтому прибегнем к дальнейшим упрощениям и воспользуемся модельной полуэллиптической плотностью состояний, предложенной в [16] (см. также [17])

$$
\begin{aligned}
\rho(\Omega) & =-\frac{1}{\pi} \operatorname{Im} G(\Omega) \\
& =\frac{1}{4 \pi t^{2}} \begin{cases}\left(16 t^{2}-\Omega^{2}\right), & |\Omega| \leq 4 t, \\
0, & |\Omega|>4 t,\end{cases} \\
\operatorname{Re} G(\Omega)= & \frac{1}{4 \pi t^{2}} \begin{cases}\Omega, & |\Omega| \leq 4 t, \\
\Omega+\left(\Omega^{2}-16 t^{2}\right)^{1 / 2}, & \Omega<-4 t, \\
\Omega-\left(\Omega^{2}-16 t^{2}\right)^{1 / 2}, & \Omega>-4 t,\end{cases}
\end{aligned}
$$

где $\Omega=\omega-\varepsilon$. По своей структуре выражения (20) и (21) относятся к одномерным структурам. 2D-характер этих выражений определяется тем, что сплошной спектр лежит в пределах $|\Omega| \leq 4 t$, а не $|\Omega| \leq 2 t$ как в 1D-случае. Отметим, что $\rho_{\max }=\rho(\Omega=0)=(\pi t)^{-1}$ и $|\operatorname{Re} G|_{\max }=|\operatorname{Re}(\Omega= \pm 4)|=(\pi t)^{-1}$.

Исходя из соотношений (15) для контакта двух одноатомных полубесконечных решеток, представим плотность состояний системы в виде

$$
\tilde{\rho}_{L(R)}=-\frac{1}{\pi} \frac{\operatorname{Re} G_{L(R)} \cdot[2]+\operatorname{Im} G_{L(R)} \cdot[1]}{[1]^{2}+[2]^{2}},
$$

где $\quad[1]=1-t^{2}\left(\operatorname{Re} G_{L} \operatorname{Re} G_{R}-\operatorname{Im} G_{L} \operatorname{Im} G_{R}\right), \quad[2]=t^{2}$ $\times\left(\operatorname{Re} G_{L} \operatorname{Im} G_{R}-\operatorname{Re} G_{R} \operatorname{Im} G_{L}\right)$ и все входящие в $(22)$ величины зависят только от энергии. 
Для моделирования функции Грина и плотности состояний полубесконечной двухатомной решетки нужно преобразовать выражения (20) и (21), заменив в них $\rho(\Omega)$ и $\operatorname{Re} G(\Omega)$ на $\rho_{A(B)}\left(\Omega_{A(B)}\right)$ и $\operatorname{Re} G_{A(B)}\left(\Omega_{A(B)}\right)$, где

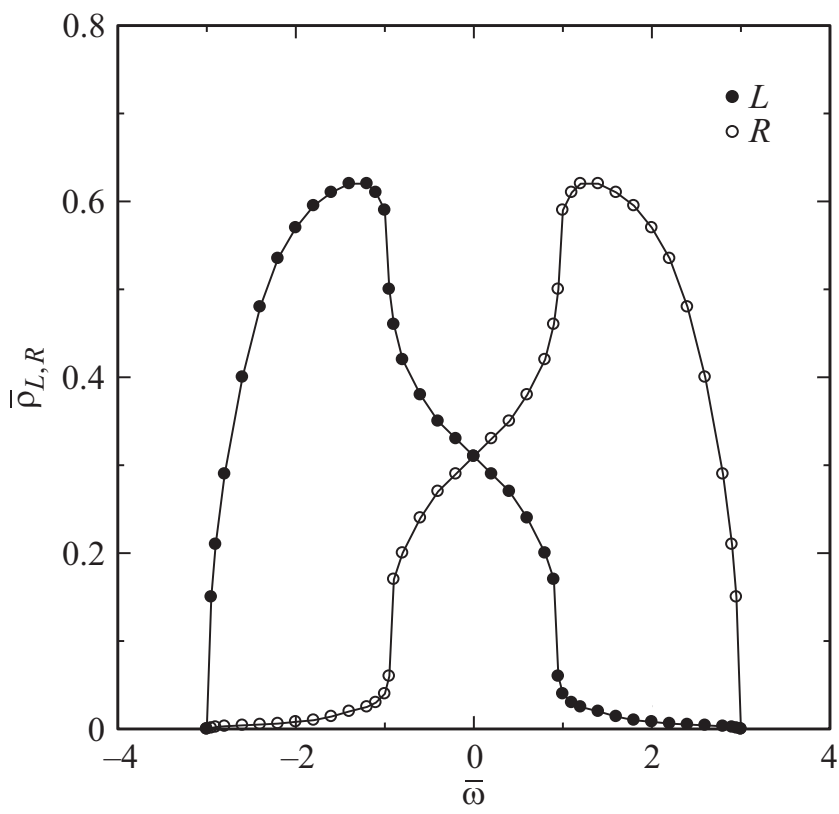

Pис. 1. Контакт одноатомных полубесконечных 2D-решеток: приведенные плотности состояний $\bar{\rho}_{L(R)}=\tilde{\rho}_{L(R)} \cdot 2 t$ в функции от безразмерной энергии $\bar{\omega}=\omega / 2 t$ при $\bar{\varepsilon}_{L}=\varepsilon_{L} / 2 t=-1$ (центр зоны проводимости левой одноатомной решетки), $\bar{\varepsilon}_{R}=\varepsilon_{R} / 2 t=1$ (центр зоны проводимости правой одноатомной решетки) и $\tau=\tilde{t} / t=1$.

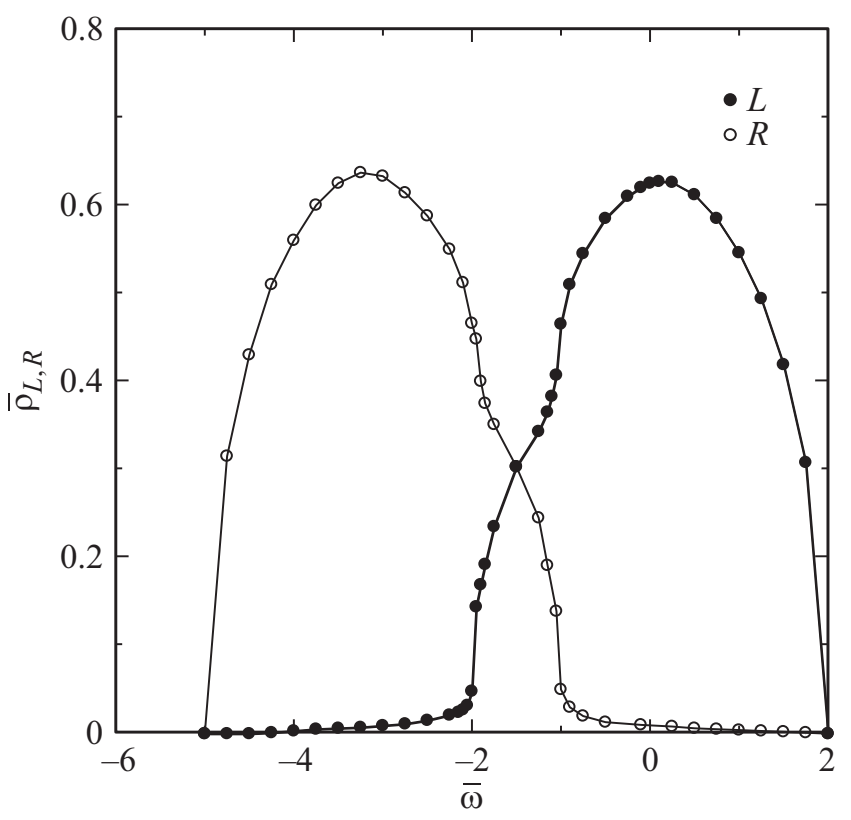

Рис. 2. Контакт одноатомной и двухатомной полубесконечных 2D-решеток: приведенные плотности состояний $\bar{\rho}_{L(R)}=\tilde{\rho}_{L A^{\prime}(R A)} \cdot 2 t \quad$ в $\quad$ функции $\quad$ от безразмерной энергии $\bar{\omega}=\omega / 2 t$ при $\bar{\varepsilon}_{L}=\varphi_{L} / 2 t=0$ (центр зоны проводимости левой одноатомной решетки), $\bar{\varepsilon}_{R}=\varepsilon_{A} / 2 t=-3$ (центр валентной зоны правой двухатомной решетки) и $\tau=\tilde{t} / t=1$.

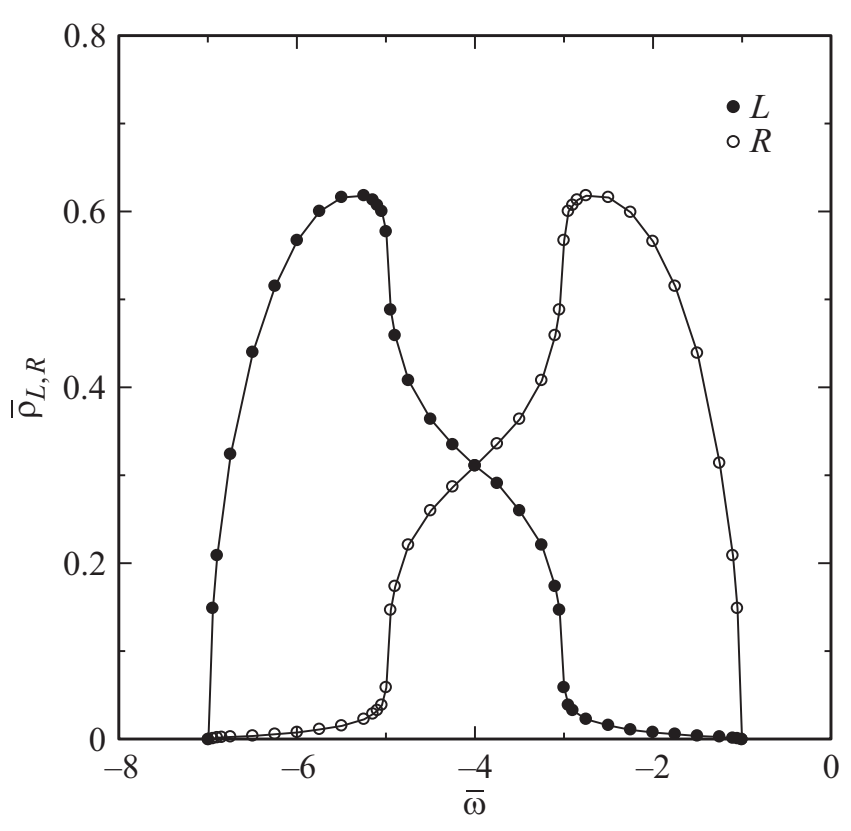

Рис. 3. Контакт двухатомных полубесконечных 2D-решеток: приведенные плотности состояний $\bar{\rho}_{L(R)}=\tilde{\rho}_{L(R)} \cdot 2 t$ в функции от безразмерной энергии $\bar{\omega}=\omega / 2 t$ при $\bar{\varepsilon}_{L}=\varepsilon_{C} / 2 t=-5$ (центр валентной зоны левой двухатомной решетки), $\bar{\varepsilon}_{R}=\varepsilon_{A} / 2 t=-3$ (центр валентной зоны правой двухатомной решетки) и $\tau=\tilde{t} / t=1$.

$\Omega_{A(B)}=\omega-\varepsilon_{A(B)}$. При этом ширина запрещенной зоны равна $E_{g}=\varepsilon_{B}-\varepsilon_{A}-8 t \quad\left(\varepsilon_{B}>\varepsilon_{A}\right)$. Затем для расчета следует воспользоваться формулами (18). Аналогичным образом для контакта двухатомных решеток в выражения (19) нужно ввести $\rho_{A(B)}\left(\Omega_{A(B)}\right), \operatorname{Re} G_{A(B)}\left(\Omega_{A(B)}\right)$ и $\rho_{C(D)}\left(\Omega_{C(D)}\right)$ и $\operatorname{Re} G_{C(D)}\left(\Omega_{C(D)}\right)$, где $\Omega_{C(D)}=\omega-\varepsilon_{C(D)}$, потребовав, чтобы $E_{g L}=\varepsilon_{D}-\varepsilon_{C}-8 t>0\left(\varepsilon_{D}>\varepsilon_{C}\right)$ и $E_{g R}=\varepsilon_{B}-\varepsilon_{A}-8 t>0\left(\varepsilon_{A(B)}>\varepsilon_{C(D)}\right)$. Подчеркнем, что в настоящей аппроксимации энергии $\varepsilon_{A(B)}$ и $\varepsilon_{C(D)}$ отвечают центрам валентных зон (зон проводимости), тогда как в формулах (2), (18), (19), (П3) и (П4) энергии $\varepsilon_{A}$ и $\varepsilon_{B}$ соответствуют краям этих зон.

Используя в качестве $\operatorname{Re} G_{L(R)}$ и $\operatorname{Im} G_{L(R)}$ выражения (20), (21), получим плотность состояний системы $\tilde{\rho}_{L(R)}(\omega)$, представленную на рис. $1-3$ для рассмотренных выше ГС и достаточно общего случая перекрытия функций $\rho_{L}(\omega)$ и $\rho_{R}(\omega)$ по энергии ${ }^{2}$. При контакте одноатомных решеток (рис. 1) и выборе начала отсчета энергии в виде $\varepsilon_{L}+\varepsilon_{R}=0$ имеем симметричную относительно нуля энергии картину, моделирующую контакт 2D-металлов.

Плотности состояний для ГС, образованной контактом одноатомной и двухатомной решеток, представлены на рис. 2 для случая, когда зона проводимости одноатомной цепочки (2D-металла) полностью перекрывает

\footnotetext{
2 Подчеркнем, что все полученные в п. 3 формулы для функций Грина и плотностей состояний, зависящих от $\omega, k_{y}$, могут быть переписаны для функций Грина и плотностей состояний, зависящих только от $\omega$
} 

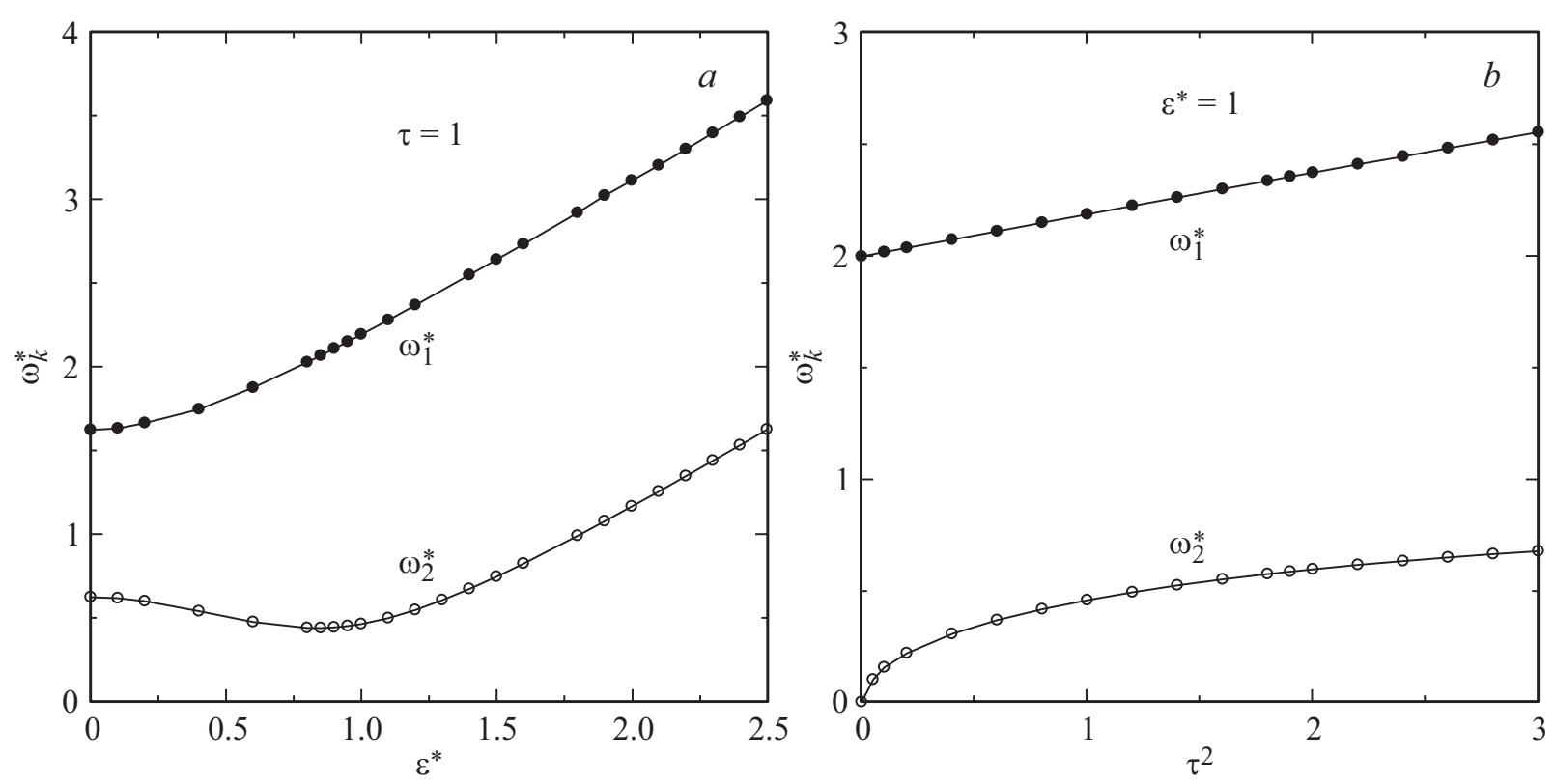

Рис. 4. Зависимость приведенных энергий уровней взаимодействующих димеров $\omega_{1,2}^{*}$ от приведенной энергии $\varepsilon^{*}$ при $\tau=1(a)$ и от квадрата безразмерной константы связи $\tau^{2}$ при $\varepsilon^{*}=1(b)$.

запрещенную зону двухатомной решетки (2D-полупроводника). При этом в силу заданной нами симметрии $\left(\varepsilon=\varepsilon_{A}+\varepsilon_{B}=0\right)$ на рис. 2 изображена только левая половина графика, относящаяся к валентной зоне двухатомной решетки. Правая половина графика представляет собой зеркальное отражение левой половины относительно нуля энергии.

Рис. 3 демонстрирует плотности состояний ГС, отвечающие контакту двухатомных решеток, моделирующему гетеропереход между двумя 2D-полупроводниками. Рассмотрен случай так называемого охватывающего (straddling) перехода $[14,15]$ с совмещенными центрами запрещенных зон. Так же как и на рис. 2, изображена только левая половина симметричного относительно нуля энергии графика, относящаяся к валентным зонам. Запрещенная зона ГС совпадает с минимальной запрещенной зоной, соответствующей правой двухатомной решетке.

Сходство графиков на рис. 1-3 обусловлено тем обстоятельством, что во всех случаях рассматриваются, по сути, контакты двух „цепочек“, функции Грина которых описываются выражениями (20), (21). Отсюда же вытекают и особенности симметрии приведенных графиков.

\section{5. Модель взаимодействующих димеров: переход заряда}

Идя по пути дальнейшего упрощения задачи, рассмотрим простейшую модель (toy model) латеральной структуры, состоящей из двух димеров: левого $(L)$, составленного из атомов с номерами -2 и -1 с энергией $s$-состояния $\varepsilon_{L}$, и правого $(R)$, образованного атомами с номерами 1 и 2 с энергией $s$-состояния $\varepsilon_{R}$. Для обоих димеров межатомная энергия перехода электрона равна $t$. Легко показать [18], что функции Грина таких димеров (в отсутствии взаимодействия между ними) равны $G_{L(R)}^{\operatorname{dim}}=g_{L(T)} / 1\left(1-t^{2} g_{L} g_{R}\right)$, где $g_{L(G)}=\left(\Omega_{L(R)}+i s\right)^{-1}$, $\Omega_{L(R)}=\omega-\varepsilon_{L(R)}$, а соответствующие плотности состояний, нормированные на два электрона $s$-орбитали, есть $\rho_{L(R)}^{\operatorname{dim}}=\delta\left(\Omega_{L(R)}-t\right)+\delta\left(\Omega_{L(R)}+t\right)$, где $\delta(\ldots)-$ дельтафункция Дирака. Включим теперь между атомами -1 и 1 взаимодействие $\tilde{t}$. Воспользовавшись уравнением Дайсона, получим функции Грина четырехатомной цепочки (см. Приложение, П3).

$$
\begin{aligned}
& G_{-2,-2}=g_{L}\left(1-g_{L}^{2} t^{2}-\frac{g_{L}^{3} g_{R} t^{2} \tilde{t}^{2}}{1-g_{R}^{2} t^{2}-g_{L} g_{R} \tilde{t}^{2}}\right)^{-1}, \\
& G_{-1,-1}=g_{L}\left(1-g_{L}^{2} t^{2}-g_{L} g_{R} \tilde{t}^{2}-\frac{g_{L} g_{R}^{3} t^{2} \tilde{t}^{2}}{1-g_{R}^{2} t^{2}}\right)^{-1},
\end{aligned}
$$

Функции Грина $G_{22}$ и $G_{11}$ получаются из выражений (23) и (24) путем замены $g_{L}$ на $g_{R}$ и $g_{R}$ на $g_{L}$. Спектр системы определяется полюсами функций Грина, которые удовлетворяют уравнению

$$
1-\left(g_{L}^{2}+g_{R}^{2}\right) t^{2}-g_{L} g_{R} \tilde{t}^{2}+g_{L}^{2} g_{R}^{2} t^{4}=0 .
$$

Корни этого уравнения приведены в Приложении, п. 3 . На рис. 4 представлены зависимости безразмерной энергий 2-х положительных уровней системы $\omega_{1,2}^{*}$ от $\varepsilon^{*}=\varepsilon / t(a)$ и $\tau^{2}(b)$; два отрицательных уровня имеют энергии $\omega_{3}^{*}=-\omega_{2}^{*}$ и $\omega_{4}^{*}=-\omega_{1}^{*}$. Особенности функций $\omega_{1,2}^{*}\left(\varepsilon^{*}, \tau^{2}\right)$ обсуждаются в п. 3 Приложения. 

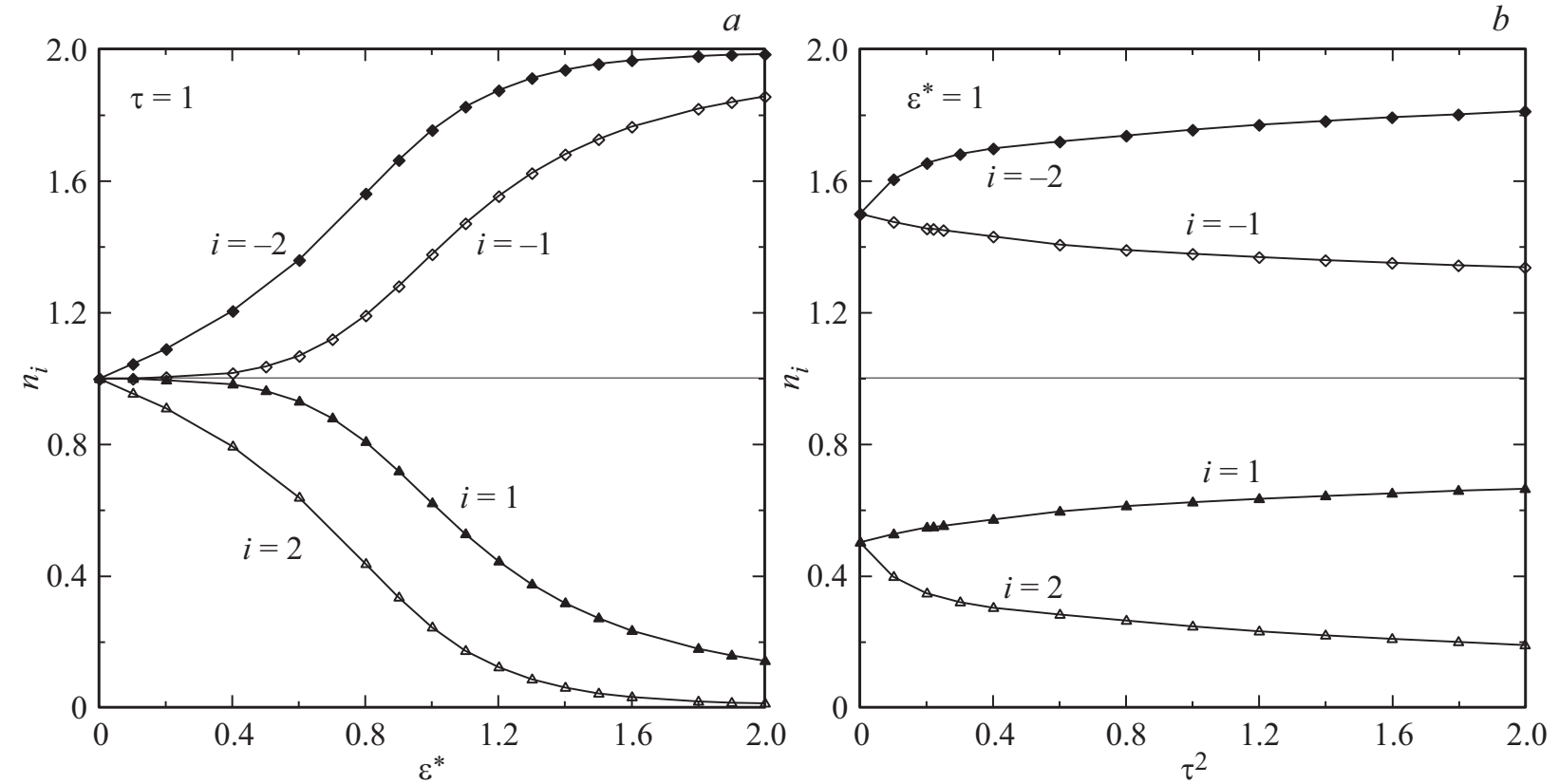

Рис. 5. Зависимости чисел заполнения $n_{i}$ атомов $i=-2,-1,1,2$ от приведенной энергии $\varepsilon^{*}$ при $\tau=1(a)$ и от квадрата безразмерной константы связи $\tau^{2}$ при $\varepsilon^{*}=1(b)$ при $-\omega_{2}^{*}<\varepsilon_{F}^{*}<\omega_{2}^{*}$.

Плотность состояний атомов четырехатомной цепочки, нормированная на два электрона, равна

$$
\rho_{i}(\omega)=\sum_{k} n_{i i}^{k} \delta\left(\omega-\omega_{k}\right),
$$

где

$$
n_{i i}^{k}=\frac{2 A_{i i}\left(\omega_{k}\right)}{\prod_{k^{\prime} \neq k}\left(\omega_{k}-\omega_{k^{\prime}}\right)},
$$

$A_{-1-1}=(\omega+\varepsilon)\left[(\omega-\varepsilon)^{2}-t^{2}\right], A_{-2-2}=(\omega-\varepsilon)^{2}(\omega+\varepsilon)$ $-\left[(\omega+\varepsilon) t^{2}+(\omega-\varepsilon) \tilde{t}^{2}\right], \quad A_{11}=(\omega-\varepsilon)\left[(\omega+\varepsilon)^{2}-t^{2}\right]$, $A_{22}=(\omega+\varepsilon)^{2}(\omega-\varepsilon)-\left[(\omega-\varepsilon) t^{2}+(\omega+\varepsilon) \tilde{t}^{2}\right]$, индексы $i=-1,-2,1,2$ нумеруют атомы, индексы $k=1,2,3$, 4 - корни уравнения (25).

На рис. 5 приведены зависимости чисел заполнения атомов $n_{i}=\int_{-\infty}^{\varepsilon_{F}} \rho_{i}(\omega) d \omega$ от параметров задачи $\varepsilon^{*}(a)$ и $\tau(b)$ в предположении, что приведенный уровень Ферми системы $\varepsilon_{F}^{*}=\varepsilon_{F} / t$ лежит в интервале $\left(-\omega_{2}^{*}, \omega_{2}^{*}\right)$, так что при нулевой температуре заполняются только уровни $-\omega_{1}^{*}$ и $-\omega_{2}^{*}$. Здесь, как и в случае, изображенном на рис. 4 (см. обсуждение в Приложении, п. 3), нулевое значение $\tau$ следует понимать как предел $\tau \rightarrow 0^{+}$. При этом число заполнения уровней атомов -2 и -1 равно 1.5, а число заполнения атомов 1 и 2 равно 0.5. Из рис. 5, $a$ ясно видно, что с ростом $\varepsilon^{*}$ заполнение атомов -2 и -1 , а 1 и 2 убывает, что вполне объясняется сдвигами уровней (рис. 4). Рис. 5, $b$ иллюстрирует нарастание заполнения атомов -2 и 1 и убыль заполнения атома -1 и 2 с ростом константы связи $\tau$. Наибольшие изменения заряда наблюдаются для крайних атомов (-2 и 2) четырехатомной цепочки, тогда как переход заряда для внутренних атомов (-1 и 1$)$ достаточно мал.

\section{6. Заключительные замечания}

В настоящей статье мы рассмотрели свободные латеральные ГС. В принципе, полученные результаты нетрудно обобщить на эпитаксиальные структуры. Для этого нужно в выражениях (1), (2), (23)-(25), (П9), (П10) добавить в знаменатели одноатомных функций Грина $g, g_{A(B)}, g_{C(D)}, g_{i i}$ сдвиги и уширения атомных уровней $\Lambda(\omega)$ и $\Gamma(\omega)$, возникающие вследствие взаимодействия контактирующих решеток с подложкой $[11,12,15,18,19]$. Воспользовавшись приведенной здесь схемой, сравнительно просто рассмотреть также контакты графеноподобных структур (как свободных, так и эпитаксиальных). При этом следует учитывать два вида контактов: зигзагообразные и крестообразные. Отметим, что для графеноподобных ГС можно воспользоваться кластерной моделью [19] аналогично тому, как в настоящей работе использовались димеры.

\section{ПРИЛОЖЕНИЕ}

1. Производя стандартную замену

$$
N_{x}^{-1} \sum_{k_{x}}(\ldots) \rightarrow \frac{a}{2 \pi} \int_{-\pi / a}^{\pi / a} d k_{x}(\ldots),
$$

для бесконечной одноатомной решетки из выражения (6) получим при $\alpha^{2}=4 t^{2} / W^{2}<1$, где 
$W=\Omega+2 t \cos \left(k_{y} \alpha\right), \Omega=\omega-\varphi, \alpha=2 t / W$ следующее выражение [20]:

$$
\begin{gathered}
G_{0}\left(\omega, m, k_{y}\right)=\frac{1}{\pi W} \int_{0}^{\pi} \frac{\cos (m x) d x}{1+\alpha \cos x}=\frac{1}{W} \frac{1}{\sqrt{1-\alpha^{2}}} I_{m}, \\
I_{m}=\left(\frac{\sqrt{1-\alpha^{2}}-1}{\alpha}\right)^{|m|} .
\end{gathered}
$$

В случае, когда $\alpha^{2}>1$ нужно заменить $\sqrt{1-\alpha^{2}}$ на $i \sqrt{\alpha^{2}-1}$.

Для бесконечной двухатомной решетки выражение для функции Грина (2) может быть переписано в виде

$$
G_{0}^{A A(B B)}(\omega)=\left(C_{A(B)}\right)^{-1} \frac{1}{1-\beta \cos \left(2 k_{x} \alpha\right)},
$$

где $\quad C_{A(B)}=g_{B(A)} Q, \quad \beta=2 t^{2} / Q \quad$ и $\quad Q=g_{A}^{-1} g_{B}^{-1}$ $-2 t^{2}\left(2+\cos \left(2 k_{y} \alpha\right)\right)$. Тогда вместо (П2) для $\beta<1$ получим

$$
\begin{aligned}
G_{0}^{A(B)}\left(\omega, m, k_{y}\right) & =\frac{1}{\pi g_{B(A)} Q} \int_{0}^{\pi} \frac{\cos (m x) d x}{1-\beta \cos x} \\
& =\frac{1}{C_{A(B)}} \frac{1}{\sqrt{1-\beta^{2}}}\left(\frac{1-\sqrt{1-\beta^{2}}}{\beta}\right)^{|m|} .
\end{aligned}
$$

При $\beta^{2}>1$ нужно заменить $\sqrt{1-\beta^{2}}$ на $i \sqrt{\beta^{2}-1}$.

2. Рассмотрим некоторые частные случаи контакта одноатомных решеток, отметив, для начала, что плотность состояний полубесконечной одноатомной квадратной решетки равна

$$
\rho_{L(R)}=-\pi^{-1} \operatorname{Im} G_{L(R)}=-(\pi t)^{-1} \sin \varphi_{L(R)} .
$$

В случае слабой связи контактирующих решеток, т.е. при $\tau^{2} \ll 1$, в нулевом порядке по $\tau^{2}$ плотность состояний (17) переходит в (П5). В первом порядке по $\tau^{2}$ имеем $\operatorname{Re} C \approx 1+\tau^{2} \cos 2 \phi$ и $\operatorname{Im} C \approx \tau^{2} \sin 2 \phi$, так что вместо (17) получим

$$
\tilde{\rho}_{L(R)} \approx \rho_{L(R)}-\left(\tau^{2} / \pi t\right) \sin \left(\varphi_{L(R)}+2 \phi\right),
$$

или $\left.\tilde{\rho}_{L} \approx \rho_{L}-\tau^{2} / \pi t\right) \sin \left(2 \varphi_{L}+\varphi_{R}\right)$ и $\left.\tilde{\rho}_{R} \approx \rho_{R}-\tau^{2} / \pi t\right)$ $\times \sin \left(2 \varphi_{R}+\varphi_{L}\right)$. Отметим, что условие $\tau^{2} \ll 1$ отвечает ван-дер-ваальсовой связи между компонентами ГС.

Положив $\varepsilon_{L}=\varepsilon_{R}=\varepsilon, \quad$ получим $\quad \varphi_{L}=\varphi_{R}=\varphi$ и $\rho=\rho_{L}=\rho_{R}, \quad$ откуда найдем $\quad \tilde{\rho}=\tilde{\rho}_{L}=\tilde{\rho}_{R} \approx$ $\approx \rho\left[1+\tau^{2}\left(3-4(\pi t) \rho^{2}\right)\right] . \quad$ При $W^{2}=4 t^{2} \quad$ плотность состояний $\rho$ максимальна и равна $\rho_{\max }=(\pi t)^{-1}$. Отсюда следует, что при том же условии плотность состояний $\tilde{\rho}$ имеет минимум, равный $\tilde{\rho}_{\min } \approx \rho\left(1-\tau^{2}\right)$. Таким образом, одногорбая плотность состояний в результате взаимодействия становится двугорбой. Это аналог возникновения связывающих и антисвязывающих состояний при взаимодействии двух уровней.
Пусть теперь $\delta \varepsilon=\varepsilon_{R}-\varepsilon_{L} \geq 8 t$. Это означает, что в интервале энергий, где $\rho_{L} \neq 0$, имеем $\rho_{R}=0$, и, наоборот, $\rho_{R} \neq 0$ в области, отвечающей $\rho_{L}=0$. Тогда $\tilde{\rho}_{L(R)} \approx \rho_{L(R)}\left\{1+\left(\tau^{2} / \pi t^{2}\right)\left[W_{R(L)}\left(W_{R(L)}-\sqrt{W_{R(I)}^{2}-4 t^{2}}\right)\right]\right\}$.

В пределе сильной связи при $\tau^{2} \gg 1$ имеем $\operatorname{Re} C \approx-\cos 2 \phi / \tau^{2}$ и $\operatorname{Im} C \approx-\sin 2 \phi / \tau^{2}$, откуда

$$
\tilde{\rho}_{L(R)} \approx \rho_{R(L)} / \tau^{2} .
$$

В этом случае следует рассматривать связанные $\tilde{t}$-взаимодействием $L$ - и $R$-края контактирующих решеток как единую систему с плотностью состояний $\tilde{\rho}_{\Sigma}=\tilde{\rho}_{L}+\tilde{\rho}_{R} \approx \rho_{\Sigma} / \tau^{2}$, где $\rho_{\Sigma}=\rho_{L}+\rho_{R}$. Подчеркнем, что предел $\tau^{2} \gg 1$ является искусственным и приведен здесь в чисто иллюстративных целях.

При $\tau=1$ получаем $\operatorname{Re} C=1 / 2$ и $\operatorname{Im} C=\operatorname{ctg} \phi / 2$, так что

$$
\tilde{\rho}_{L(R)}=\left(\rho_{R(L)}-\cos \varphi_{L(R)} \operatorname{ctg} \phi / \pi t\right) / 2 .
$$

При $\varepsilon_{L}=\varepsilon_{R}=\varepsilon$ получим $\varphi_{L}=\varphi_{R}=\varphi, \rho=\rho_{L}=\rho_{R}$ и $\tilde{\rho}_{L}=\tilde{\rho}_{R}=\tilde{\rho}$, откуда $\tilde{\rho}=1 / 2(\pi t)^{2} \rho$. Для случая $\delta \varepsilon=\varepsilon_{R}-\varepsilon_{L} \geq 8 t \quad$ учтем, в области, где $\rho_{L} \neq 0$, $\rho_{R}=0 \quad$ имеем $\quad \operatorname{ctg} \phi=\operatorname{ctg}\left(\varphi_{L} / 2\right) ; \quad$ в области, где $\rho_{L}=0, \quad \rho_{R} \neq 0 \quad$ имеем $\quad \operatorname{ctg} \phi=\operatorname{ctg}\left(\varphi_{R} / 2\right) . \quad$ Тогда $\tilde{\rho}_{L(R)}=\left[\rho_{L(R)}-\cos \varphi_{L(R)} \operatorname{ctg}\left(\varphi_{L(R)} / 2\right) / \pi t\right] / 2$. Отметим, что условие $\tau=1$ отвечает ситуации, когда расстояние между атомами краев левой и правой подрешеток равно $a$.

3. Для четырехатомной цепочки, образованной двумя димерами, введем функции Грина $G_{i j}$, где индексы $i$ и $j$ пробегают значения $-2,-1,1$ и 2 . Воспользуемся уравнением Дайсона $G=g+g \hat{T} G$, где $g_{i j}=g_{i i} \delta_{i j}$, $g_{-2-2}=g_{-1-1}=g_{L}, g_{11}=g_{22}=g_{R}, \delta_{i j}-$ символ Кронекера, $\hat{T} \hat{T}^{+}=\tilde{t}^{2}$ для взаимодействия атомов -1 и 1 и в $\hat{T} \hat{T}^{+}=\tilde{t}^{2}$ остальных случаях. В результате получим

$$
\begin{aligned}
G_{-2-2}= & g_{-2-2}\left(1-g_{-2-2} g_{-1-1} t^{2}\right. \\
& \left.-\frac{g_{-2-2} g_{-1-1}^{2} g_{11} t^{2} \tilde{t}^{2}}{1-g_{11} g_{22} t^{2}-g_{11} g_{-1-1} \tilde{t}^{3}}\right)^{-1} \\
G_{-1-1}= & g_{-1-1}\left(1-g_{-2-2} g_{-1-1} t^{2}-g_{-1-1} g_{11} \tilde{t}^{2}\right. \\
& \left.-\frac{g_{-1-1} g_{11}^{2} g_{22} t^{2} \tilde{t}^{2}}{1-g_{11} g_{22} t^{2}}\right)^{-1}
\end{aligned}
$$

Функции Грина $G_{22}$ и $G_{11}$ получаются из выражений (П9) и (П10) путем замены знаков индексов $i j$ на противоположные. Переходя от $g_{i j}$ к функциям Грина $g_{L}$ и $G_{R}$, получим выражения (23) и (24).

Проанализируем теперь спектр системы, определяемый корнями уравнения (25), для чего положим $-\varepsilon_{L}=\varepsilon_{R}=\varepsilon>0$. Тогда уравнение (П11) сводится к уравнению $\omega^{4}-2 \omega^{2} b+c=0$, где $b=\varepsilon^{2}+t^{2}+\tilde{t}^{2} / 2$, $c=\left(\varepsilon^{2}-t^{2}\right)^{2}+\varepsilon^{2} \tilde{t}^{2}$. Это уравнение имеет четыре решения: $\omega_{1,2}^{*}=\sqrt{b^{*} \pm R^{*}}$ и $\omega_{4,3}^{*}=-\sqrt{b^{*} \pm R^{*}}$, где $\omega_{k}^{*}=$ $=\omega_{k} / t \quad(k=1,2,3,4), R^{*}=\sqrt{4 \varepsilon^{*^{2}}+\tau^{2}\left(1+\tau^{2} / 4\right)}$, 
$b^{*}=b / t^{2}$ и $\varepsilon^{*}=\varepsilon / t$. Легко показать, что $\left(b^{*}\right)^{2}-\left(R^{*}\right)^{2}=$ $=\left(\left(\varepsilon^{*}\right)^{2}-1\right)^{2}+\tau^{2}\left(\varepsilon^{*}\right)^{2} \geq 0$.

На рис. 3 демонстрируется зависимость приведенных энергий $\omega_{1,2}^{*}$ от $\varepsilon^{*}=\varepsilon / t \quad(a)$ и $\tau^{2}(b)$. Как следует из рис. 3 , функции $\omega_{1}^{*}\left(\varepsilon^{*}\right)$ и $\omega_{1,2}^{*}\left(\tau^{2}\right)$ являются монотонно возрастающими, тогда как функция $\omega_{2}^{*}\left(\varepsilon^{*}\right)$ имеет минимум. Такая зависимость имеет место только при значениях $\tau^{2}$, удовлетворяющих неравенству $4 \geq \tau^{2}\left(1+\tau^{2} / 4\right)$; значение $\varepsilon^{*}$, отвечающее минимуму, есть $\left(\varepsilon^{*}\right)_{\min }=\left[4-\tau^{2}\left(1+\tau^{2} / 4\right)\right]^{1 / 2} / 2$. При $\varepsilon^{*} \rightarrow \infty$ получаем $\omega_{1,2} \rightarrow \sqrt{\left(\varepsilon^{*}\right)^{2} \pm \varepsilon^{*}}$; при $\tau \rightarrow \infty$ имеем $\omega_{1}^{*} \propto \tau$, $\omega_{2}^{*} \propto \varepsilon^{*}$. Нулевое значение $\tau$ (рис. $3, b$ ) следует понимать как предел $\tau \rightarrow 0^{+}$, что отвечает взаимодействующим с бесконечно малой константой связи димерам. При этом, согласно правилам квантовой механики, состояния изолированных димеров, соответствующие приведенным энергиям +1 и -1 , группируются в четыре состояния с безразмерными энергиями $+2,-2$ и 0 (дважды вырожденное состояние).

\section{Список литературы}

[1] A.K. Geim, I.V. Grigorieva. Nature 499, 419 (2013).

[2] H. Wang, F. Liu, W. Fu, Z. Fang, W. Zhou, Z. Liu Nanoscale 6, 12250 (2014).

[3] И.В. Антонова. ФТП 50, 67 (2016).

[4] J. Wang, F. Ma, M. Sun. RSC Adv. 7, 16801 (2017).

[5] Q. Sun, Y. Dai, Y. Ma, N. Yin, W. Wei, L. Yu, B. Huang. 2D Materials 3, 035017 (2016).

[6] J. Zhang, W. Xie, X. Xu, S. Zhang, J. Zhao. Chem. Mater 28, 5022 (2016).

[7] Y. Zhao, Z. Wan, X. Xu, S.R. Patil, U. Hetmaniuk, M.P. Anantram. Sci. Rep. 5, 10712 (2015).

[8] S. Bruzzone, D. Logoteta, G. Fiori, G. Iannaccone. Sci. Rep. 5, 14519 (2015).

[9] G.C. Loh, R. Pandey. J. Mater. Chem. C 3, 5918 (2015).

[10] Z.-Y. Ong, G. Zhang, Y.-W. Zhang. Phys. Rev. B 93, 075406 (2016).

[11] С.Ю. Давыдов, А.А. Лебедев, О.В. Посредник. Элементарное введение в теорию наносистем. Лань, СПб (2014). $192 \mathrm{c}$.

[12] С.Ю. Давыдов. Теория адсорбции: метод модельных гамильтонианов. Изд-во СПбГЭТУ ЛЭТИ, СПб (2013). 235 c.; twirpx.com/file/1596114/

[13] D. Kalkstein, P. Soven. Surf. Sci. 26, 85 (1971).

[14] Ф. Бехштедт, Р. Эндерлайн. Поверхности и границы раздела полупроводников. Мир, М. (1990). 488 с.

[15] С.Ю. Давыдов. ФТТ 58, 1182 (2016).

[16] D.M. Newns. Phys. Rev. 178, 1123 (1969).

[17] T.L. Einstein, J.R. Schrieffer. Phys. Rev. B 7, 8, 3629 (1973).

[18] С.Ю. Давыдов. ФТТ 54, 2193 (2012).

[19] С.Ю. Давыдов. ФТТ 59, 1650 (2017).

[20] И.С. Градштейн, И.М. Рыжик. Таблицы интегралов, сумм, рядов и произведений. Наука, М. (1971). 1108 с.

\section{Редактор Т.Н. Василевская}

